\title{
Double-detonation sub-Chandrasekhar supernovae: can minimum helium shell masses detonate the core?
}

\author{
M. Fink, F. K. Röpke, W. Hillebrandt, I. R. Seitenzahl, S. A. Sim, and M. Kromer
}

\author{
Max-Planck-Institut für Astrophysik, Karl-Schwarzschild-Str. 1, 85741 Garching, Germany \\ e-mail: mfink@mpa-garching.mpg.de
}

Received 16 December 2009 / Accepted 8 February 2010

\begin{abstract}
The explosion of sub-Chandrasekhar mass white dwarfs via the double detonation scenario is a potential explanation for type Ia supernovae. In this scenario, a surface detonation in a helium layer initiates a detonation in the underlying carbon/oxygen core leading to an explosion. For a given core mass, a lower bound has been determined on the mass of the helium shell required for dynamical burning during a helium flash, which is a necessary prerequisite for detonation. For a range of core and corresponding minimum helium shell masses, we investigate whether an assumed surface helium detonation is capable of triggering a subsequent detonation in the core even for this limiting case. We carried out hydrodynamic simulations on a co-expanding Eulerian grid in two dimensions assuming rotational symmetry. The detonations are propagated using the level-set approach and a simplified scheme for nuclear reactions that has been calibrated with a large nuclear network. The same network is used to determine detailed nucleosynthetic abundances in a post-processing step. Based on approximate detonation initiation criteria in the literature, we find that secondary core detonations are triggered for all of the simulated models, ranging in core mass from 0.810 up to $1.385 M_{\odot}$ with corresponding shell masses from 0.126 down to $0.0035 M_{\odot}$. This implies that, as soon as a detonation triggers in a helium shell covering a carbon/oxygen white dwarf, a subsequent core detonation is virtually inevitable.
\end{abstract}

Key words. supernovae: general - nuclear reactions, nucleosynthesis, abundances - hydrodynamics - methods: numerical

\section{Introduction}

The basic physical mechanism for type Ia supernova (SN Ia) explosions has become widely accepted ever since it was first proposed almost 50 years ago (see e.g. Hillebrandt \& Niemeyer 2000, for a review): a thermonuclear explosion in electrondegenerate matter (Hoyle \& Fowler 1960) produces radioactive ${ }^{56} \mathrm{Ni}$ that, by its decay, releases the energy that powers the light curve (Truran et al. 1967; Colgate \& McKee 1969; Kuchner et al. 1994). Despite this long history, the questions of the progenitor system and the explosion scenario have not been completely answered. In the so-called double detonation sub-Chandrasekhar model, a detonation in an accreted helium shell causes a secondary detonation of a carbon/oxygen white dwarf (C/O WD) core (e.g. Woosley \& Weaver 1994; Livne \& Arnett 1995). This happens at a total mass below the Chandrasekhar limit and can lead to a wide range of possible explosion strengths. Recent population synthesis studies suggest that such events are in principle frequent enough to account for a significant part of the observed SN Ia rate (Belczynski et al. 2005; Ruiter et al. 2009) ${ }^{1}$.

This scenario hinges on two critical points - first the formation of a detonation in the helium shell and second whether a successful detonation of the helium shell can detonate the core. Here we focus on the second question. A secondary detonation can be triggered in two different ways: either directly when the

\footnotetext{
${ }^{1}$ But note that there is still some discrepancy between these predictions and those made from observations, which tend to be significantly lower (Deloye et al. 2005; Bildsten et al. 2007).
}

helium detonation shock hits the core/shell interface ("edge-lit"), or with some delay, after the shock has converged near the center. The edge-lit case is more restrictive since it requires a strong shock wave and might only work if the helium detonation starts at some altitude above the base of the shell (cf. Livne \& Glasner 1990). In this work we examine the delayed mechanism since it may still lead to a core detonation even for shocks too weak for the edge-lit case. In this spirit of determining minimal conditions for a core detonation, we look into models with helium shell masses that are substantially lower than previously considered. Provided a detonation is triggered in these, the shock they drive into the core is expected to be particularly weak.

Fink et al. (2007, hereafter referred to as Paper I) found a secondary core detonation to be robustly triggered in multidimensional hydrodynamic simulations of generic $1-M_{\odot}$ models with shell masses of 0.1 to $0.2 M_{\odot}$. Here we revisit the doubledetonation sub-Chandrasekhar supernova scenario for a series of models with different core-shell mass combinations. We use the minimum helium shell masses required for dynamical runaway calculated by Bildsten et al. (2007). We find that even for this most conservative case, a helium detonation initiated at the base of the shell robustly triggers a secondary detonation in the $\mathrm{C} / \mathrm{O}$ core. We therefore also investigate the hydrodynamic evolution, nucleosynthesis, and observational implications of such explosions.

Section 2 contains the setup of our simulations. In Sect. 3 we describe the hydrodynamics and nuclear reaction network codes used to perform the simulations. The results are presented in 
detail in Sect. 4. Section 5 discusses the results and their observational consequences in an astrophysical context. Our work is summarized in Sect. 6.

\section{Explosion scenario}

\subsection{Initial models}

The models we study are WDs with a composition of ${ }^{12} \mathrm{C}$ and ${ }^{16} \mathrm{O}$ (equal parts by mass) with minimum accreted helium shell mass $\left(M_{\mathrm{sh}}\right)$ that might lead to a detonation, as calculated by Bildsten et al. (2007). They assumed a certain $M_{\text {core }}-M_{\text {sh }}$ combination and calculated the resulting structure and $P_{\mathrm{b}}, T_{\mathrm{b}}$ evolution (with $M_{\text {core }}$ being the mass of the accretor WD, and $P_{\mathrm{b}}$ and $T_{\mathrm{b}}$ being the pressure and temperature at the base of the shell) in a series of hydrostatic equilibrium integrations assuming a constant-temperature core of $T_{\text {core }}=3 \times 10^{7} \mathrm{~K}$ and a fully convective shell with an adiabatic temperature profile. Heating by the triple- $\alpha$ reaction in a pure helium shell was taken into account. Thus, the maximum temperatures during the flash could be determined and also the shortest nuclear burning timescales. In series of calculations with varying $M_{\mathrm{sh}}$, the minimum flash ignition masses that allow for a dynamical burning event and potential detonation $M_{\min }$ were determined (the term dynamical refers to the condition that the nuclear burning timescale at the shell base, where it is hottest, becomes as short as the local dynamical timescale).

Bildsten et al. (2007) also study AM Canum Venaticorum binaries consisting of a $\mathrm{C} / \mathrm{O}$ WD accreting at high rates from a helium white dwarf donor. They show that for WD masses $\gtrsim 0.8 M_{\odot}$ flash masses reach or surpass $M_{\min }$ before the donor mass is depleted. This makes these systems interesting candidates for the sub-Chandrasekhar scenario as they might be frequent and also might explode at small helium shell masses $\left(0.01-0.1 M_{\odot}\right)$.

The initial models for our hydro simulations were constructed as follows: given $\rho_{\mathrm{c}}$ (central density), $T_{\text {core }}, \rho_{\mathrm{b}}$, and $T_{\mathrm{b}}$ at the instant when burning becomes dynamical (provided by Bildsten and Shen, private communication), hydrostatic equilibrium was integrated using our own code's equation of state. It differs slightly from Bildsten et al. (2007), e.g. it does not include Coulomb corrections to the ion pressure. Therefore, our core/shell masses might slightly differ, but this should not change the main properties of the explosion dynamics.

The most important parameters of our initial models are given in Table 1. In our models we neglect any potential enhancements of the chemical composition due to the metallicity of the progenitors. The core masses $M_{\text {core }}$ range from $\sim 0.8 M_{\odot}$ for Model 1 to nearly the Chandrasekhar mass for Model 6 . As already mentioned, the shell masses $M_{\text {sh }}$ are set to $M_{\min }$. The density at the base of the shell varies only moderately between 3.7 and $8.7 \times 10^{5} \mathrm{~g} \mathrm{~cm}^{-3}$.

\subsection{Detonation initiation}

The simulations are carried out in a similar manner as in the previous study (Paper I). In all models, the helium shell is ignited at a single point at its base. This one-point ignition scenario introduces an asymmetry which makes it harder to trigger a core detonation than in symmetric shell-ignited models. Moreover, it is expected to introduce viewing angle effects in the synthetic light curves and spectra. As the simulation is carried out in $2 \mathrm{D}$ rotational symmetry, the ignition spot is placed on the positive $z$-axis of the cylindrical coordinate system.
Table 1. Parameters of our initial models.

\begin{tabular}{ccccccc}
\hline \hline Model & 1 & 2 & 3 & 4 & 5 & 6 \\
\hline$M_{\text {tot }}$ & 0.936 & 1.004 & 1.080 & 1.164 & 1.293 & 1.3885 \\
$M_{\text {core }}$ & 0.810 & 0.920 & 1.025 & 1.125 & 1.280 & 1.3850 \\
$M_{\text {sh }}$ & 0.126 & 0.084 & 0.055 & 0.039 & 0.013 & 0.0035 \\
$\rho_{\mathrm{c}, 7}$ & 1.45 & 2.4 & 4.15 & 7.9 & 28 & 140 \\
$\rho_{\mathrm{b}, 5}$ & 3.7 & 4.0 & 4.5 & 6.1 & 6.4 & 8.7 \\
\hline
\end{tabular}

Notes. $\rho_{\mathrm{c}, 7}$ denotes the central density in units of $10^{7} \mathrm{~g} \mathrm{~cm}^{-3}, \rho_{\mathrm{b}, 5}$ is the density at the base of the helium shell in units of $10^{5} \mathrm{~g} \mathrm{~cm}^{-3} \cdot M_{\text {tot }}, M_{\text {core }}$, and $M_{\mathrm{sh}}$ are the masses of the WD, the $\mathrm{C} / \mathrm{O}$ core, and the helium shell, respectively. All masses are given in solar masses.

We follow the detonations in the helium shell and in the WD core with two separate level set functions (see also Sect. 3.2). The helium detonation is started by hand by setting the first level set function to positive values in a volume of choice at the base of the shell. Since dynamical burning is a necessary but not sufficient criterion for the initiation of a detonation, the formation of the helium detonation is a fundamental assumption in our models.

After shell ignition, the WD core is scanned for "hot spots" arising from the shock exerted on the core by the helium detonation. If a sufficiently large volume becomes hot and dense enough, a $\mathrm{C} / \mathrm{O}$ detonation is initiated by setting the second level set function to positive values in this whole volume ${ }^{2}$. To decide when to initiate the detonation, critical densities and temperatures given in the literature are used (Niemeyer \& Woosley 1997; Röpke et al. 2007; see Tables 1 and 2 of Paper I).

These detonation initiation criteria, which are based on the assumption of spherically symmetric linear temperature gradients extending into unburned fuel, are limited by some inherent uncertainties (e.g. Seitenzahl et al. 2009 point out that the functional form of temperature and density gradients of the hot spot significantly affects the critical detonation initiation conditions). Furthermore, the process depends on the composition of the fuel, the background temperature and local velocity fields ${ }^{3}$. Since the critical length scales pertinent to detonation initiation are generally much smaller than the grid resolution, addressing these details is currently impossible in our full-star simulations. However, the detonation initiations in this work are fairly robust: the critical temperatures and densities we apply are significantly exceeded (see Table 2). Fully resolved calculations of the detonation initiation conditions are expected to only moderately change the critical conditions. Thus, our conclusions should hold despite these uncertainties.

\section{Numerical simulation}

Our numerical hydrodynamics scheme, briefly summarized below in Sect. 3.1, is similar to the one used in Paper I. However, there have been major changes to the treatment of the burning physics, which are described in Sect. 3.2. Also, a post-processing scheme is now employed to determine more realistic nucleosynthetic yields (Sect. 3.3).

\footnotetext{
2 To prevent grid geometry effects on the flame, every hot cell initiates a burning bubble with a radius of three cells around it.

${ }^{3}$ For such effects, volume burning behind the converging shock wave would have to be accounted for, but this is not yet implemented in our code.
} 
M. Fink et al.: Double-detonation sub-Chandrasekhar supernovae: can minimum helium shell masses detonate the core?

Table 2. Conditions at core detonation initiation for all models.

\begin{tabular}{ccccccc}
\hline \hline Model & $t_{\text {ign }}[\mathrm{s}]$ & $T_{9, \text { ign }}$ & $\rho_{8 \text {,ign }}$ & $z_{\text {ign }}[\mathrm{km}]$ & $\left|z_{\text {ign }}\right| / R_{\text {core }}$ & $\Delta[\mathrm{km}]$ \\
\hline 1 & 1.94 & 4.62 & 1.08 & -1910 & 0.39 & 9.86 \\
2 & 1.78 & 4.15 & 1.58 & -1850 & 0.40 & 8.43 \\
3 & 1.58 & 4.42 & 2.24 & -1750 & 0.41 & 6.81 \\
4 & 1.30 & 4.09 & 1.89 & -1560 & 0.41 & 5.59 \\
5 & 1.00 & 4.14 & 4.63 & -1370 & 0.46 & 3.86 \\
6 & 0.64 & 3.23 & 8.92 & -1040 & 0.50 & 2.54 \\
\hline
\end{tabular}

Notes. $t_{\text {ign }}, T_{9, \text { ign }}, \rho_{8, \text { ign }}$, and $z_{\text {ign }}$ are the time, temperature in $10^{9} \mathrm{~K}$, density in $10^{8} \mathrm{~g} \mathrm{~cm}^{-3}$, and $z$ coordinate at the ignition spot, respectively. $R_{\text {core }}$ denotes the radius of the C/O-WD core and $\Delta$ the grid resolution, which is approximately $1 / 1000$ of the WD radius.

\subsection{Hydrodynamics}

In our Eulerian hydrodynamics code the reactive Euler equations are solved using a finite volume scheme based on the PROMETHEUS code by Fryxell et al. (1989), which is an implementation of the "piecewise parabolic method" of Colella \& Woodward (1984). In order to track the expanding WD during the explosion, a co-expanding uniform grid as in Röpke \& Hillebrandt (2005), and Röpke (2005) is used ${ }^{4}$. In some of the simulations (Models 4-6) exponentially growing cell sizes (e.g. Reinecke et al. 2002b; Röpke et al. 2006) are used in the outer parts of the grid in order to keep a sufficiently high resolution in the $\mathrm{C} / \mathrm{O} \mathrm{WD}$ during the helium shell detonation and successive shell expansion. The grid resolution was $1024 \times 2048$ cells in $2 \mathrm{D}$ rotational symmetry. An equation of state for WD matter and monopole gravity complete the system. The equation of state includes contributions of an arbitrarily degenerate and arbitrarily relativistic electron gas, nuclei that follow a MaxwellBoltzmann distribution, photons, and electron/positron pairs. The dependency on changes in the number of electrons per baryon, $Y_{\mathrm{e}}$, is also taken into account.

\subsection{Nuclear burning}

The two detonations are followed by independent level sets in passive implementation (Osher \& Sethian 1988; Reinecke et al. 1999; Golombek \& Niemeyer 2005). The burning velocity is calculated as a function of the local density: in the ${ }^{4} \mathrm{He}$ detonation the Chapman-Jouguet case is assumed and the burning speed relative to the ashes is the local sound speed. In $\mathrm{C} / \mathrm{O}$ at high densities above $\sim 2 \times 10^{7}$ self-sustained detonations are of pathological type (e.g. Sharpe 1999). For the burning speed relative to the fuel the values of Gamezo et al. (1999) are used above $10^{7} \mathrm{~g} \mathrm{~cm}^{-3}$. At lower densities the Chapman-Jouguet case is assumed.

A simplified scheme is used to model the energy release of nuclear reactions, similar to Paper I and Reinecke et al. (2002a): the code includes five species variables for ${ }^{4} \mathrm{He},{ }^{12} \mathrm{C},{ }^{16} \mathrm{O}$, intermediate mass elements (IMEs), and iron group elements (IGEs). Changes in composition and internal energy due to fast reactions occur instantaneously behind the flame discontinuity. To this end, the final abundances and $Q$-values have been tabulated against fuel density. This new prescription constitutes an improvement to the burning scheme of Paper I. Details are given in the Appendix.

\footnotetext{
4 The expansion of a fixed mass shell is tracked. To resolve the shock convergence arising from the helium detonation, for Models 1-3 the grid is kept static until the end of helium burning and for models 4-6 it is kept static until the onset of $\mathrm{C} / \mathrm{O}$ detonation.
}

\subsection{Post-processing}

Our post-processing scheme is similar to Travaglio et al. (2004): passively advected tracer particles are used in the hydro runs and detailed nucleosynthetic yields are calculated afterwards from their density and temperature trajectories. To this end, a large nuclear network with 384 species ranging from protons, neutrons, and $\alpha$-particles to ${ }^{98}$ Mo is employed. A description of the code used to solve the large nuclear network can be found in Thielemann et al. (1990), Thielemann et al. (1996), and Iwamoto et al. (1999). The reaction rate libraries adopted in this work are the same as in those references, however, newer tables for weak reaction rates (Langanke \& Martinez-Pinedo 2000) were used as described in Travaglio et al. (2004).

A number of $n_{\text {core }}=80 \times 160=12800$ tracer particles is placed equidistant in radial mass coordinate and $\cos \theta$ in the WD core such that each particle represents the same mass ${ }^{5}$. To spatially resolve the nucleosynthesis in the thin helium shell despite its lower mass, the same number of $n_{\text {sh }}=n_{\text {core }}$ tracer particles was placed there. With this distribution, particles in the core and in the shell represent masses of $\frac{M_{\text {core }}}{n_{\text {core }}}$ and $\frac{M_{\mathrm{sh}}}{n_{\mathrm{sh}}}$, respectively.

\section{Simulation results}

In this section we present the main simulation results. All the models behave in a characteristically similar manner. Therefore our discussion will focus on Model $2\left(M_{\text {core }}=0.92, M_{\mathrm{sh}}=\right.$ $0.084 M_{\odot}$ ) as a detailed example but we will also comment on the other models where appropriate.

\subsection{Explosive evolution}

Starting from the ignition point (see top-left panel of Fig. 1), the helium detonation surrounds the whole core until it converges again at the "south pole". While the detonation moves along the base of the shell, an oblique shock wave propagates into the core, converging off-center at a point on the negative $z$-axis (cf. Livne \& Glasner 1990; Paper I) ${ }^{6}$. As can be seen in Fig. 1, this resembles the self-similar problem of a spherically or cylindrically converging shock wave (cf. Guderley 1942; Landau \& Lifschitz 1991; Ponchaut et al. 2006), which results in strong shock compression if sufficiently small scales are resolved. Due to self-similarity, the scales that need to be resolved to reach a certain compression just become smaller if the shock from the shell detonation is weaker. However, the maximum possible compression is limited (cf. Paper I). The question to be addressed in this study is whether the volume at which high enough temperatures and densities for dynamical carbon burning are achieved is large enough for a detonation to form. In our simulations we always reached densities $>10^{8} \mathrm{~g} \mathrm{~cm}^{-3}$ and temperatures $>3.2 \times 10^{9} \mathrm{~K}$ on resolved scales $>2.5 \mathrm{~km}$. The critical radius for detonation formation at these conditions is, however, only $2 \mathrm{~m}$. Thus, the critical conditions for detonation initiation are met for all our models, despite small shell masses. Table 2

\footnotetext{
5 An offset is added to the coordinates such that each particle has a random initial position within its corresponding fluid element.

6 Rotation effects that are neglected here could break the symmetry of the one-point ignition scenario if the ignition spot was located off the rotation axis. This could distort the minimum shocked volume and make a core detonation more difficult. The spherical shell ignition case and all ignition geometries that are symmetric with respect to the rotation axis should, however, not be influenced.
} 


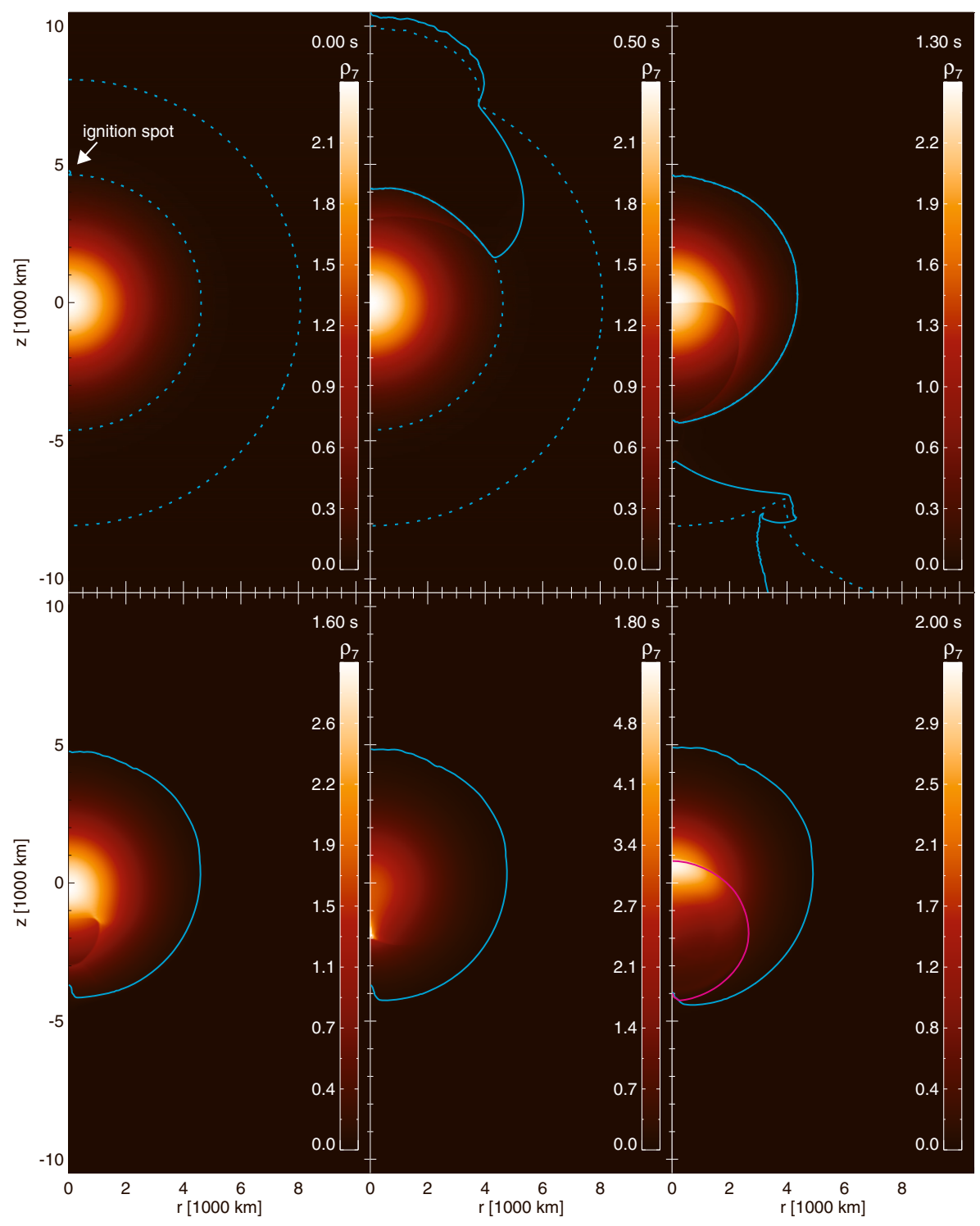

Fig. 1. Explosion evolution for Model 2. The density is color coded, and the solid cyan and magenta lines are the locations of the helium and $\mathrm{C} / \mathrm{O}$ detonation flames, respectively. Dashed lines in cyan mark the border of the helium shell. lists the conditions at which the core detonations were ignited. As in Paper I, a conservative critical temperature of $4 \times 10^{9} \mathrm{~K}$ was used. Thus, the given values are only a lower limit for the maximum possible compression at the given grid resolution $\Delta$. If the detonation would have been suppressed, stronger compression would have been achieved. Only Model 6 did not surpass $4 \times 10^{9} \mathrm{~K}$, despite being simulated at the highest spatial resolution. The conditions reached in the shock compression, however, were still sufficient to safely assume a successful detonation triggering. Therefore, it was ignited at this lower temperature in a second run. This model verifies that it is harder to compress the core sufficiently if the helium shell mass is small. Conversely, the high initial density of the most massive model makes a detonation easier. Based on our approximate initiation criteria we conclude that the limiting factor for a successful core detonation is only the successful formation of a detonation in the helium shell.

Table 2 gives times and positions of the detonation initiations on the $z$-axis. That the carbon detonations occur earlier for smaller shell masses can be explained by the decrease of the core radii associated with the increasing core masses. At a smaller radius the helium detonation has a shorter way around the core while the helium detonation speed at the base of the shell is roughly constant for all models. Note that the approximately self-similar nature of the problem results in the curious fact that the ignition spots of the core detonations lie at similar relative distances $\left(0.4-0.5 R_{\text {core }}\right)$ from the center.

We will now discuss our fiducial case (Model 2) in detail. At densities $\$ 4 \times 10^{5} \mathrm{~g} \mathrm{~cm}^{-3}$ in the helium shell, burning is relatively incomplete and nuclear statistical equilibrium (NSE) is not reached. The final composition is $\sim 63 \%$ of ${ }^{4} \mathrm{He}, \sim 10 \%$ of IMEs, and $\sim 26 \%$ of IGEs (see Sect. 4.2 and Table 3 for more details). The C/O detonation starts at $t \sim 1.8 \mathrm{~s}$ and at $z \sim-1900 \mathrm{~km}$ (see Fig. 1) and it produces $0.34 M_{\odot}$ of ${ }^{56} \mathrm{Ni}, 0.44 M_{\odot}$ of IMEs and $0.11 M_{\odot}$ of ${ }^{16} \mathrm{O}$. The structure of the ejecta at $t=10 \mathrm{~s}$, where our simulations stop and the ejecta are close to homologous expansion, is shown in Fig. 2. The shown distribution has been derived from the tracer particles after the post-processing step. Due to the two detonations, IGEs can be found both in the central region and in the shell. The shock of the $\mathrm{C} / \mathrm{O}$ detonation partially penetrates into the helium-detonation ashes. This gives rise to the Richtmyer-Meshkov instability which generates vortices at the boundary between $\mathrm{C} / \mathrm{O}$ - and helium-detonation ashes (see upper right part of Fig. 2). This effect causes some mixing between unburned $\mathrm{C} / \mathrm{O}$ and IGEs from the helium detonation. 
M. Fink et al.: Double-detonation sub-Chandrasekhar supernovae: can minimum helium shell masses detonate the core?

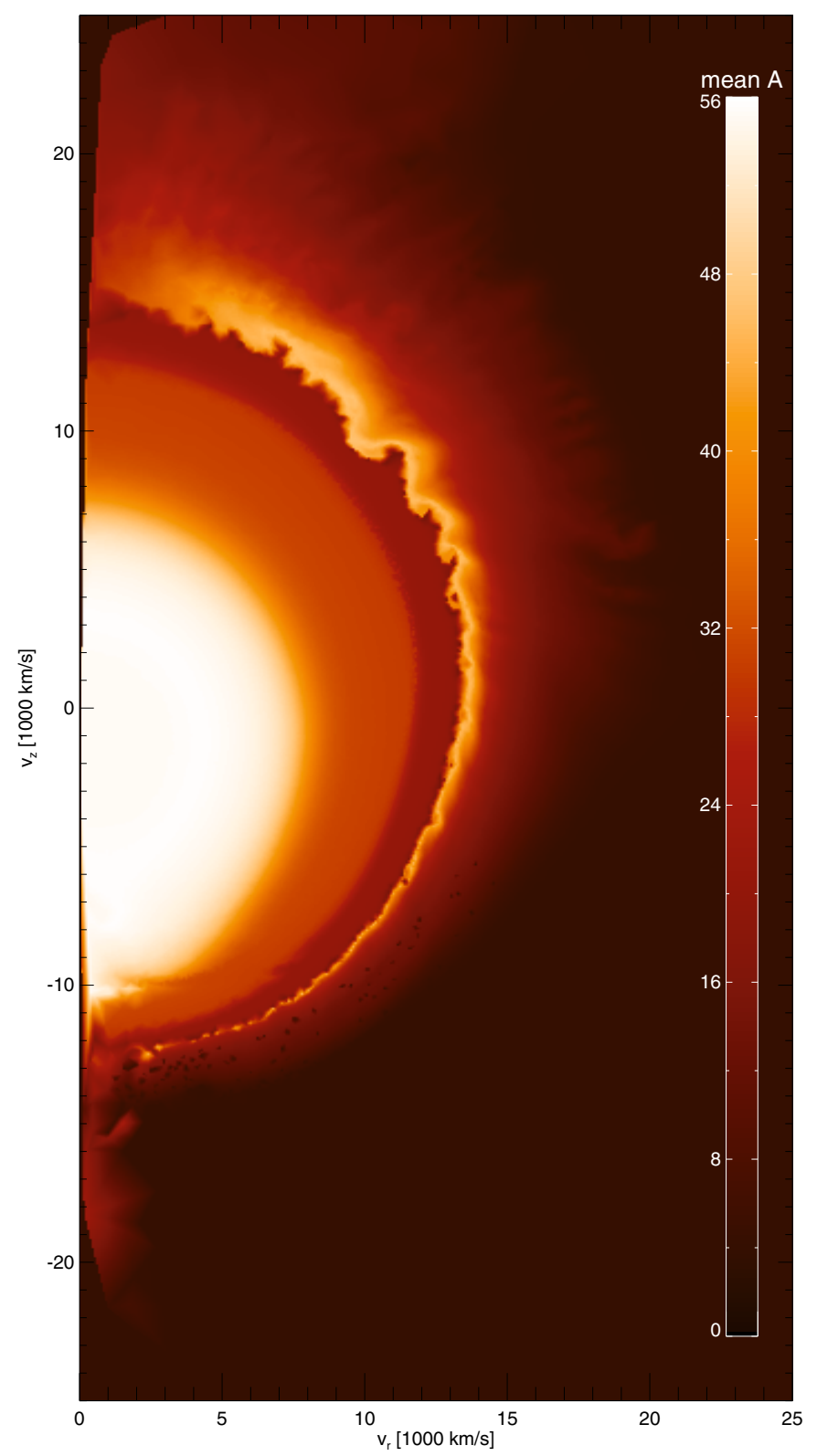

Fig. 2. Ejecta structure in velocity space for Model 2 at $10 \mathrm{~s}$. Color coded is the mean mass number (averaged over mass: $\bar{A}=\sum_{i} A_{i} X_{i}$, where $A_{i}$ and $X_{i}$ are mass number and mass fraction of a nucleus $i$, respectively).

\subsection{Nucleosynthesis}

The total nucleosynthetic yields of the explosions are given in Table 3 for each model. The upper part lists the results of the $\mathrm{C} / \mathrm{O}$ core detonation, whereas the lower shows those for the helium shell detonation. Neutronization becomes important only for the highest mass model showing $16 \%$ of IGEs that are not ${ }^{56} \mathrm{Ni}$. This model is also peculiar in having almost no IMEs (only $\sim 2 \%$ of the total mass). The NSE freeze-out is $\alpha$-rich for all models showing significant contributions of ${ }^{4} \mathrm{He},{ }^{57} \mathrm{Ni},{ }^{58} \mathrm{Ni}$, and ${ }^{60} \mathrm{Zn}$ in the final composition. Figure 3 shows the distribution of the nucleosynthetic products in velocity space along the equatorial axis (this is representative of the mean for the whole explosion).

A slice of Fig. 2 at the $z=0$ plane gives an overview of all nucleosynthesis taking place in Model 2 (Fig. 4). Products of the $\mathrm{C} / \mathrm{O}$ detonation are located below velocities of $\sim 13000 \mathrm{~km} \mathrm{~s}^{-1}$.

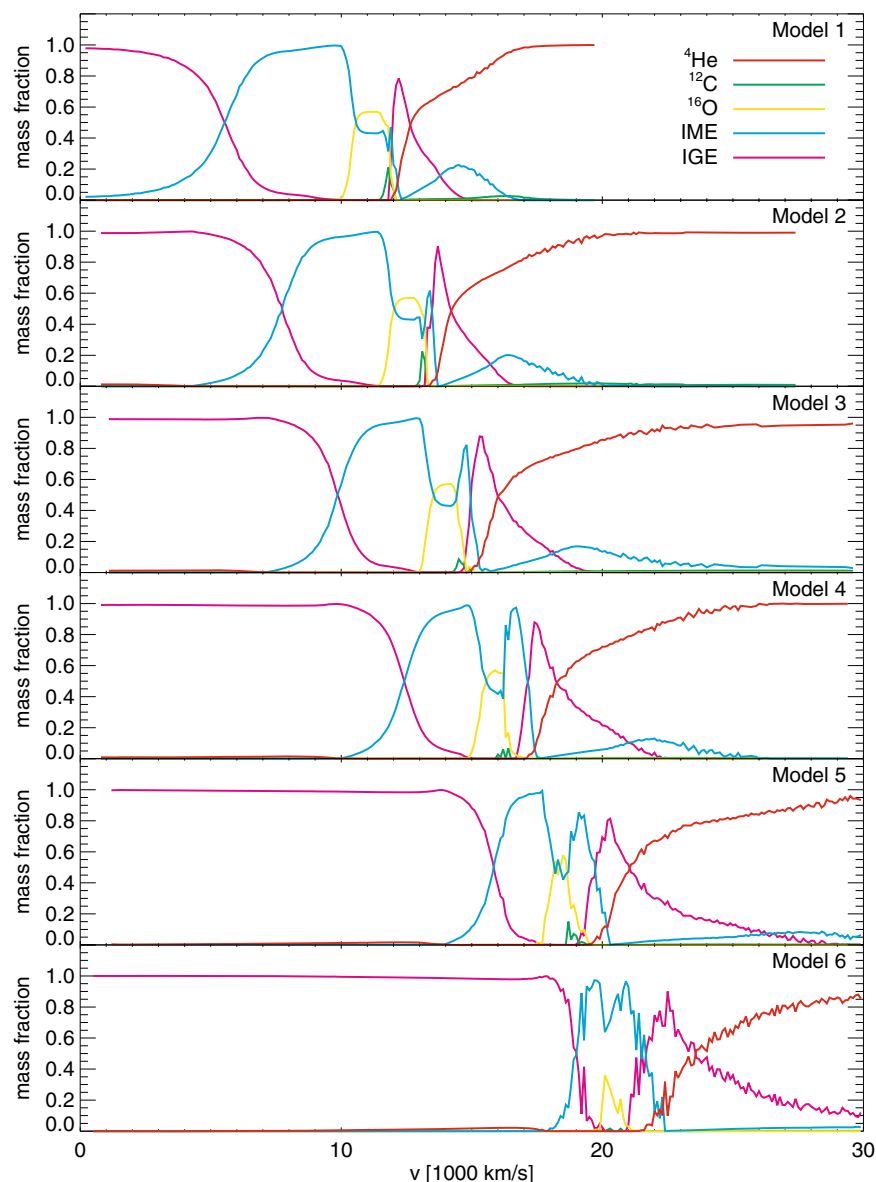

Fig. 3. Mass fractions of the main groups of nuclei in velocity space for all models at $10 \mathrm{~s}$. Shown is the average of an angle range of $\pm \pi / 32$ around the equator. The velocity bin size is $100 \mathrm{~km} \mathrm{~s}^{-1}$.

The yields are: IGEs: $39 \%$, IMEs: $48 \%,{ }^{16} \mathrm{O}: 12 \%$ (see also Table 3). For the low central densities present in most of the models, relatively large amounts of IMEs and ${ }^{16} \mathrm{O}$ are found. However, there is almost no unburned ${ }^{12} \mathrm{C}$.

The helium detonation products are located at $v \gtrsim 13000 \mathrm{~km} \mathrm{~s}^{-1}$ above the $\mathrm{C} / \mathrm{O}$ detonation layers. As discussed by Bildsten et al. (2007), the burning products at those low densities differ significantly from previously published values (e.g. Khokhlov 1984, 1989). For Model 2 the most abundant nuclei are unburned ${ }^{4} \mathrm{He}(63 \%)$, the IGEs ${ }^{48} \mathrm{Cr}(9 \%),{ }^{52} \mathrm{Fe}(8 \%)$, and ${ }^{44} \mathrm{Ti}(6 \%)$, and the IMEs ${ }^{40} \mathrm{Ca}(4 \%),{ }^{36} \mathrm{Ar}(4 \%)$, and ${ }^{32} \mathrm{~S}(1 \%)$. The low-density helium burning regime is characterized by not reaching NSE and the fact that at the low maximum temperatures $\alpha$-captures are much faster than the triple- $\alpha$ reactions. For the low initial densities in the outer shell, helium is mostly unburned. Deeper in the shell the higher densities increase the triple- $\alpha$ rate meaning that more ${ }^{4} \mathrm{He}$ is burned and higher maximum temperatures are reached. Once a ${ }^{12} \mathrm{C}$ nucleus is formed, $\alpha$-captures process it rapidly to higher mass numbers. Since the Coulomb barriers increase with mass number, this process stops at some maximum mass number depending on the local temperature. This leads to an inwards increase of the mean mass number, peaking roughly at ${ }^{48} \mathrm{Cr}$ or ${ }^{52} \mathrm{Fe}$. The final yields of Model 2 in velocity space (Figs. 4 and 5) clearly demonstrate this trend. In the very innermost parts of the shell, where the initial densities are highest, the mass numbers are again lower. This is due to enrichment by carbon produced by triple- $\alpha$ reactions that take place before the onset of the detonation. 
Table 3. Total nucleosynthetic yields of selected species or groups of species.

\begin{tabular}{|c|c|c|c|c|c|c|}
\hline Model & 1 & 2 & 3 & 4 & 5 & 6 \\
\hline & \multicolumn{6}{|c|}{$\mathrm{C} / \mathrm{O}$ core detonation } \\
\hline$M_{\mathrm{C} / \mathrm{O}, \text { fuel }}$ & $8.1 \times 10^{-1}$ & $9.2 \times 10^{-1}$ & 1.03 & 1.13 & 1.28 & 1.39 \\
\hline$M_{\mathrm{IGES}}$ & $1.8 \times 10^{-1}$ & $3.6 \times 10^{-1}$ & $5.7 \times 10^{-1}$ & $8.2 \times 10^{-1}$ & 1.11 & 1.33 \\
\hline$M_{\mathrm{IMES}}$ & $4.8 \times 10^{-1}$ & $4.4 \times 10^{-1}$ & $3.7 \times 10^{-1}$ & $2.6 \times 10^{-1}$ & $1.2 \times 10^{-1}$ & $3.1 \times 10^{-2}$ \\
\hline$M_{56 \mathrm{Ni}}$ & $1.7 \times 10^{-1}$ & $3.4 \times 10^{-1}$ & $5.5 \times 10^{-1}$ & $7.8 \times 10^{-1}$ & 1.05 & 1.10 \\
\hline$M_{52 \mathrm{Fe}}$ & $7.6 \times 10^{-3}$ & $9.9 \times 10^{-3}$ & $9.6 \times 10^{-3}$ & $7.9 \times 10^{-3}$ & $4.2 \times 10^{-3}$ & $1.7 \times 10^{-3}$ \\
\hline$M_{48} \mathrm{Cr}$ & $3.9 \times 10^{-4}$ & $4.6 \times 10^{-4}$ & $4.5 \times 10^{-4}$ & $3.8 \times 10^{-4}$ & $2.1 \times 10^{-4}$ & $7.1 \times 10^{-5}$ \\
\hline$M_{16 \mathrm{O}}$ & $1.4 \times 10^{-1}$ & $1.1 \times 10^{-1}$ & $8.0 \times 10^{-2}$ & $4.2 \times 10^{-2}$ & $3.1 \times 10^{-2}$ & $1.2 \times 10^{-2}$ \\
\hline \multirow[t]{2}{*}{$M_{12 \mathrm{C}}$} & $6.6 \times 10^{-3}$ & $4.4 \times 10^{-3}$ & $2.7 \times 10^{-3}$ & $8.8 \times 10^{-4}$ & $5.9 \times 10^{-3}$ & $7.4 \times 10^{-4}$ \\
\hline & \multicolumn{6}{|c|}{ Helium shell detonation } \\
\hline$M_{\mathrm{He}, \text { fuel }}$ & $1.3 \times 10^{-1}$ & $8.4 \times 10^{-2}$ & $5.5 \times 10^{-2}$ & $3.9 \times 10^{-2}$ & $1.3 \times 10^{-2}$ & $3.5 \times 10^{-3}$ \\
\hline$M_{\mathrm{IGEs}}$ & $2.9 \times 10^{-2}(23 \%)$ & $2.2 \times 10^{-2}(26 \%)$ & $1.7 \times 10^{-2}(30 \%)$ & $1.3 \times 10^{-2}(33 \%)$ & $4.2 \times 10^{-3}(32 \%)$ & $1.1 \times 10^{-3}(31 \%)$ \\
\hline$M_{\mathrm{IMES}}$ & $1.3 \times 10^{-2}(10 \%)$ & $8.2 \times 10^{-3}(10 \%)$ & $5.3 \times 10^{-3}(10 \%)$ & $5.7 \times 10^{-3}(15 \%)$ & $1.9 \times 10^{-3}(14 \%)$ & $7.3 \times 10^{-4}(21 \%)$ \\
\hline$M_{56 \mathrm{Ni}}$ & $8.4 \times 10^{-4}(1 \%)$ & $1.1 \times 10^{-3}(1 \%)$ & $1.7 \times 10^{-3}(3 \%)$ & $4.4 \times 10^{-3}(11 \%)$ & $1.5 \times 10^{-3}(11 \%)$ & $5.7 \times 10^{-4}(16 \%)$ \\
\hline$M_{52} \mathrm{Fe}$ & $7.6 \times 10^{-3}(6 \%)$ & $7.0 \times 10^{-3}(8 \%)$ & $6.2 \times 10^{-3}(11 \%)$ & $3.5 \times 10^{-3}(9 \%)$ & $1.2 \times 10^{-3}(10 \%)$ & $2.0 \times 10^{-4}(6 \%)$ \\
\hline$M_{48} \mathrm{Cr}$ & $1.1 \times 10^{-2}(9 \%)$ & $7.8 \times 10^{-3}(9 \%)$ & $4.4 \times 10^{-3}(8 \%)$ & $2.2 \times 10^{-3}(6 \%)$ & $6.8 \times 10^{-4}(5 \%)$ & $1.5 \times 10^{-4}(4 \%)$ \\
\hline$M_{44} \mathrm{Cr}_{\mathrm{Ti}}$ & $7.9 \times 10^{-3}(6 \%)$ & $5.4 \times 10^{-3}(6 \%)$ & $3.4 \times 10^{-3}(6 \%)$ & $1.8 \times 10^{-3}(5 \%)$ & $4.9 \times 10^{-4}(4 \%)$ & $6.2 \times 10^{-5}(2 \%)$ \\
\hline$M_{40}{ }^{11}$ & $4.7 \times 10^{-3}(4 \%)$ & $3.2 \times 10^{-3}(4 \%)$ & $2.2 \times 10^{-3}(4 \%)$ & $2.2 \times 10^{-3}(6 \%)$ & $6.8 \times 10^{-4}(5 \%)$ & $2.4 \times 10^{-4}(7 \%)$ \\
\hline$M_{4} \mathrm{He}$ & $8.4 \times 10^{-2}(66 \%)$ & $5.3 \times 10^{-2}(63 \%)$ & $3.3 \times 10^{-2}(60 \%)$ & $2.0 \times 10^{-2}(52 \%)$ & $6.9 \times 10^{-3}(54 \%)$ & $1.7 \times 10^{-3}(48 \%)$ \\
\hline$M_{L}$ & $2.0 \times 10^{-1}$ & $3.7 \times 10^{-1}$ & $5.7 \times 10^{-1}$ & $8.0 \times 10^{-1}$ & 1.06 & 1.10 \\
\hline$E_{\text {kin }}\left[10^{51} \mathrm{erg}\right]$ & 0.90 & 1.04 & 1.20 & 1.40 & 1.59 & 1.68 \\
\hline
\end{tabular}

Notes. $M_{\mathrm{C} / \mathrm{O} \text {, fuel }}$ and $M_{\mathrm{He} \text {, fuel }}$ are the total masses of initial fuel in the $\mathrm{C} / \mathrm{O}$ core and the helium shell, respectively. For the helium detonation the values in brackets give the fraction of an isotope mass to the total shell mass $M_{\mathrm{He} \text {, fuel }} . M_{\mathrm{L}}$ is the total mass of all radioactive species that could power a light curve: ${ }^{56} \mathrm{Ni},{ }^{52} \mathrm{Fe}$, and ${ }^{48} \mathrm{Cr}$. All masses are given in units of $M_{\odot}$. $E_{\text {kin }}$ is the asymptotic total kinetic energy.

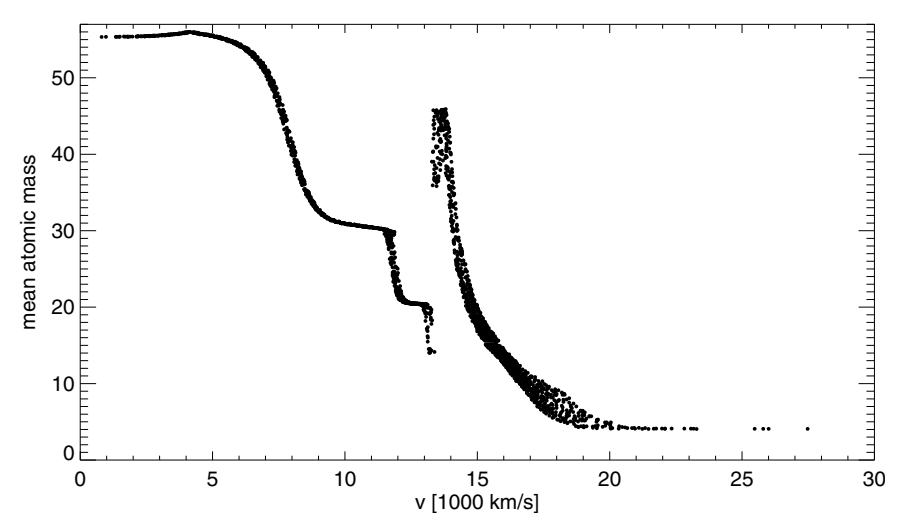

Fig. 4. Mean atomic mass number in the equatorial plane $(z=0)$ for Model 2 at $10 \mathrm{~s}$. Points give the values for individual tracer particles within $\theta=\pi \pm \pi / 32$.

This occurs because of the high temperatures at the base of the helium shells in our initial models. In a mixture of ${ }^{12} \mathrm{C}$ and ${ }^{4} \mathrm{He}$, if the carbon mass fraction $X_{{ }_{12} \mathrm{C}}$ exceeds a value of $\frac{12}{A}$, then there is not enough ${ }^{4} \mathrm{He}$ to form nuclei with mass number $A$ or higher by $\alpha$-captures in the course of a detonation passing through this matter. E.g., for $X_{{ }^{12} \mathrm{C}} \geq \frac{1}{3}$ one expects $A_{\max } \leq 36$.

The initial triple- $\alpha$ burning in the hottest regions of the shell in the initial models provided by Bildsten et al. (2007) has not been taken into account in our hydrodynamic simulations, as they lack a nuclear network that could calculate this volume burning effect. It has, however, been considered in our postprocessing step. This introduces some asymmetry in the results which reflects our choice of a pure helium shell composition and single spot ignition: while the detonation wave wraps around the WD, more and more ${ }^{12} \mathrm{C}$ is produced in the remaining shell material. For Model 2, the mass-fraction of initial ${ }^{12} \mathrm{C}$ which is reached at the equator by this volume burning is consistent with

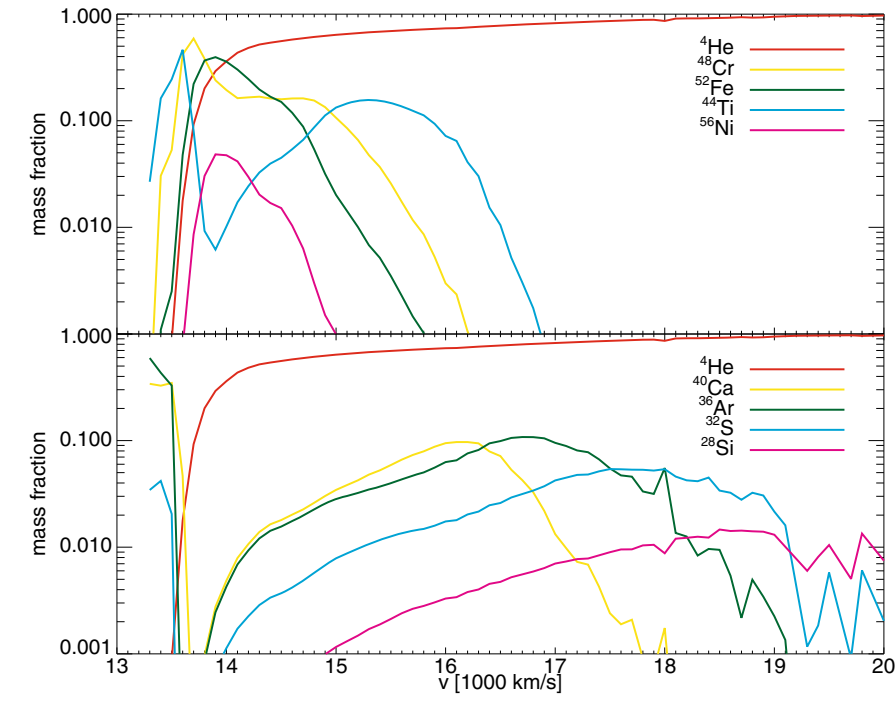

Fig. 5. Distribution of the isotopes in the ejecta in velocity space for Model 2 at $10 \mathrm{~s}$. Shown is the average of an angle range of $\pm \pi / 32$ around the equator. The velocity bin size was $100 \mathrm{~km} \mathrm{~s}^{-1}$.

the minimum mass fraction values given in Shen \& Bildsten (2009). Above the equatorial plane there is less initial carbon, below there is more. Therefore our total yields should be roughly consistent with spherically symmetric or one-dimensional simulations based on their initial compositions. The values published in Bildsten et al. (2007) from the one-dimensional detonation of a similar model, however, differ from ours as they assume a detonation in pure ${ }^{4} \mathrm{He}$. Thus it is not surprising that they reach ${ }^{56} \mathrm{Ni}$ by $\alpha$-captures and find it to be the most abundant burning product. The fact that they burn $\sim 57 \%$ of the helium and we only about $40 \%$ (in Model 3, which is closest to their model) could 
M. Fink et al.: Double-detonation sub-Chandrasekhar supernovae: can minimum helium shell masses detonate the core?

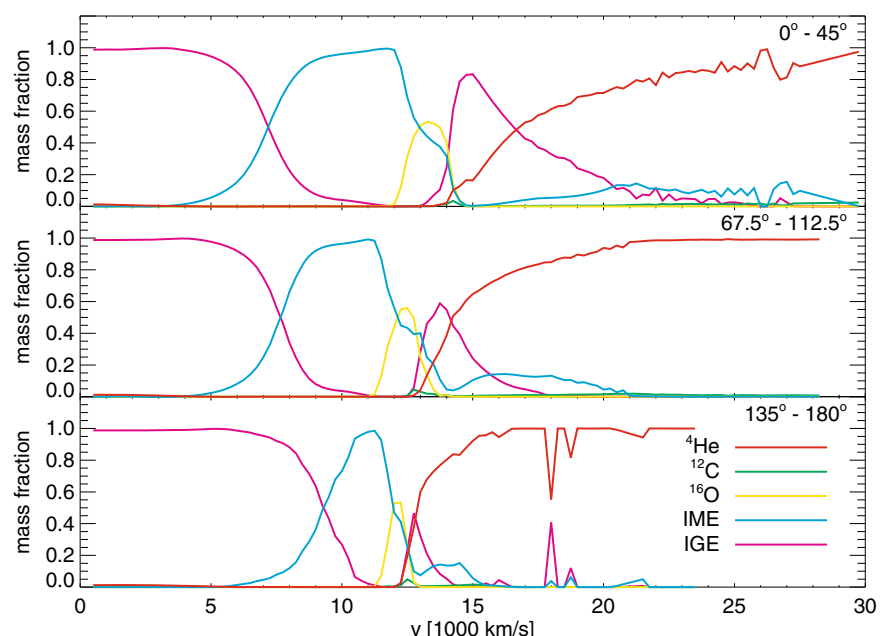

Fig. 6. Asymmetry of mass fractions in velocity space for Model 2 at $10 \mathrm{~s}$. (Group) abundances are averaged for three different polar angle ranges and over velocity bins of $250 \mathrm{~km} \mathrm{~s}^{-1}$.

be due to the different explosion dynamics of our one-point ignited and their whole-shell ignited detonation.

\subsection{Asymmetry effects}

In Fig. 2 asymmetries in both composition and ejecta velocities of Model 2 are visible. These are quantified in Fig. 6, which shows the abundances of the main groups of elements averaged for three different angular ranges: $0^{\circ}-45^{\circ}$ ("north"), $67.5^{\circ}-112.5^{\circ}$ ("equator"), and $135^{\circ}-180^{\circ}$ ("south"). The main differences are:

- Helium detonation products in the north extend over a wider velocity range than those in the south. As the ejecta are already homologous to very good accuracy at $10 \mathrm{~s}$, this cannot change by stronger expansion of the southern ejecta. The effect is also visible in the final distribution of tracer particles shown in Fig. 7. As every particle represents the same mass, it can also be seen that the southern regions are denser than the northern ones. This property might be important for spectrum formation, as a wider range in velocity space increases the range of possible photon absorption frequencies.

- From north to south the amount of burned material decreases. It is $48 \%, 37 \%$, and $24 \%$, for the northern, equatorial, and southern directions of Fig. 6, respectively. Also the amount of synthesized IGEs is less (corresponding to lower maximum mass numbers in Fig. 2): it is $88 \%, 66 \%$, and $51 \%$ of the burned matter, respectively.

- At the south the $\mathrm{C} / \mathrm{O}$ core has been compressed more strongly: the outer boundary of the core ejecta is found to be roughly at $14000 \mathrm{~km} \mathrm{~s}^{-1}, 13000 \mathrm{~km} \mathrm{~s}^{-1}$, and $12000 \mathrm{~km} \mathrm{~s}^{-1}$ from the top panel to the bottom panel of Fig. 6. This is also consistent with the IGE abundance from the core detonation increasing from north to south $(30 \%, 36 \%, 55 \%)$ at the expense of IMEs $(56 \%, 50 \%, 38 \%)$ and oxygen.

- While the core ejecta extend to lower velocities in the south the IGEs reach significantly higher velocities there.

- Especially in the northern hemisphere there seems to be significant mixing of unburned carbon and oxygen from the core with IGEs from the shell detonation. This might be due to the Richtmyer-Meshkov instability (see Sect. 4.1).

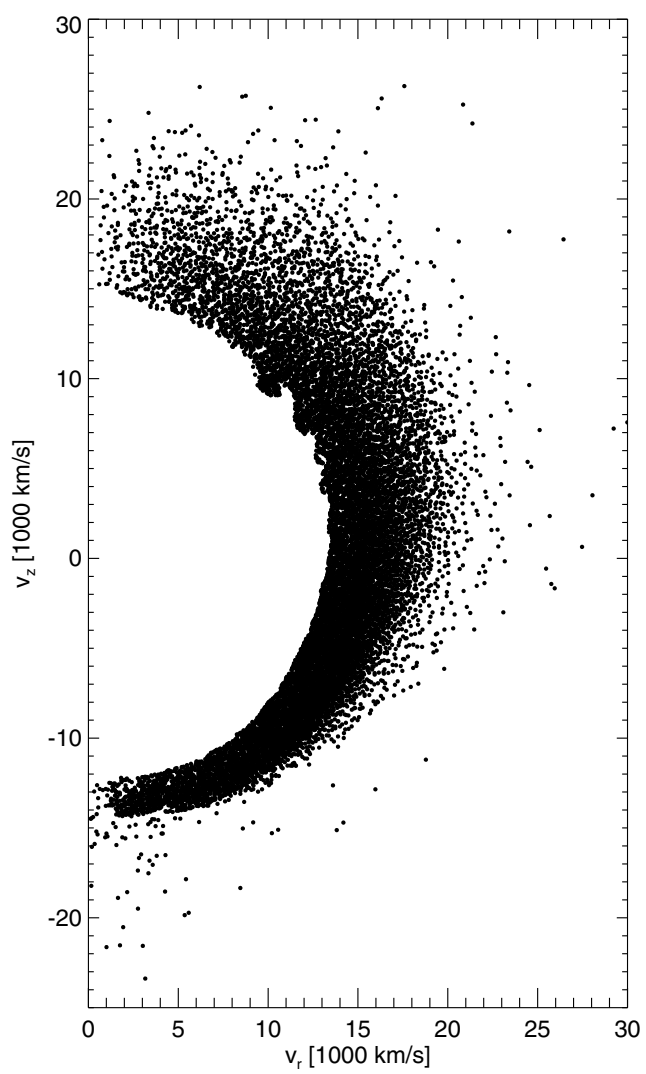

Fig. 7. Distribution of tracer particles in velocity space for Model 2 at $10 \mathrm{~s}$. Shown are only particles which initially belong to the helium shell.

Qualitatively similar asymmetry effects appear for the other models of our study.

\section{Discussion}

The primary goal of this work has been to investigate whether or not secondary core detonations can be triggered for scenarios with the minimum helium shell masses of Bildsten et al. (2007). We find that secondary core detonation conditions leading to a successful explosion of the WD are obtained in all of our six simulations. We now comment on the key observational implications of our double-detonation models.

Depending on the initial central density of the model, the $\mathrm{C} / \mathrm{O}$ detonation produces nickel masses between 0.17 and $1.1 M_{\odot}$. In principle, this range is sufficiently broad to encompass all major classes of type Ia supernovae (cf., e.g., Stritzinger et al. 2006, for a sample of ${ }^{56} \mathrm{Ni}$ masses determined for 16 well-observed SNe Ia): the high mass end of subluminous SN $1991 \mathrm{bg}^{7}$-like events $\left(\sim 0.07-0.17 M_{\odot}\right.$ : Model 1), normal type Ia supernovae $\left(\sim 0.4-0.8 M_{\odot}\right.$ : Models $\left.2-4\right)$, and bright SN $1991 \mathrm{~T}^{8}$-like explosions ( 0.85-1.0 $M_{\odot}$ : Models 4, 5). In Fig. 8 we show angle-averaged ultraviolet-optical-infrared (UVOIR) light curves for all six of our models (computed with the ARTIS code; Kromer \& Sim 2009; Sim 2007; corresponding values are given in Table 4). The light curves illustrate that not only does the peak magnitude vary significantly between the models, as expected from the differences in nickel mass, but that there is also significant diversity in both the rise times

\footnotetext{
7 Filippenko et al. (1992); Leibundgut et al. (1993).

8 Phillips et al. (1992).
} 


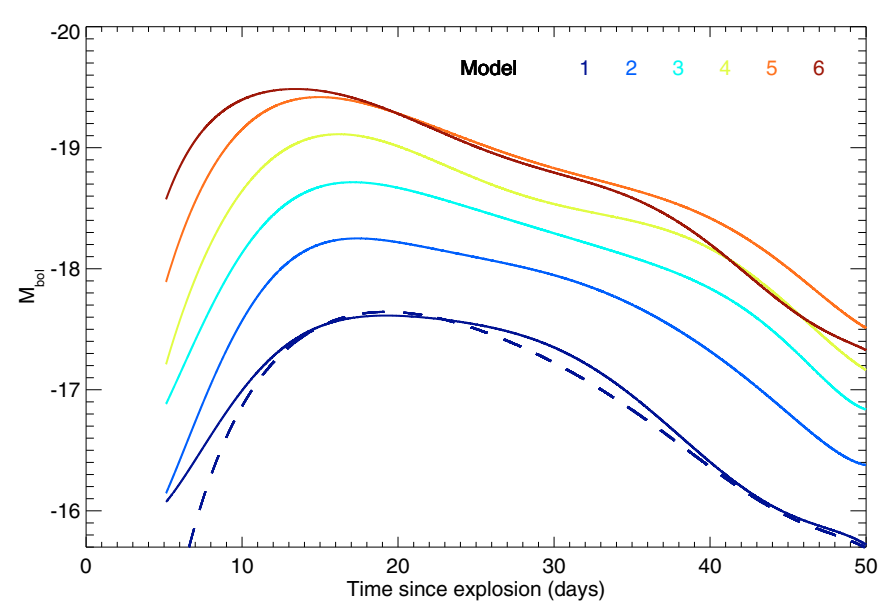

Fig. 8. UVOIR bolometric light curves for the models. Two light curves are shown for Model 1: the first (solid line) shows the calculation for the complete model while the second (dashed line) shows the result obtained when the contributions of radioactive ${ }^{52} \mathrm{Fe}$ and ${ }^{48} \mathrm{Cr}$ are neglected.

Table 4. Rise times $t_{\text {peak }}$ and peak absolute magnitudes $M_{\text {peak }}^{\text {bol }}$ for the UVOIR bolometric light curves of the models.

\begin{tabular}{ccccccc}
\hline \hline Model & 1 & 2 & 3 & 4 & 5 & 6 \\
\hline$t_{\text {peak }}[$ days $]$ & 18.6 & 18.6 & 18.0 & 15.5 & 14.4 & 13.9 \\
$M_{\text {peak }}^{\text {bol }}$ & -17.6 & -18.2 & -18.7 & -19.1 & -19.4 & -19.5 \\
$\Delta m_{15}^{\text {bol }}$ & 0.64 & 0.40 & 0.50 & 0.55 & 0.55 & 0.66 \\
\hline
\end{tabular}

Notes. $\Delta m_{15}^{\text {bol }}$ is the change in bolometric magnitude between maximum light and 15 days thereafter. Due to Monte Carlo noise magnitudes are uncertain by $\sim 0.1 \mathrm{mag}$.

(see Table 4) and the post-maximum light curve shape. This diversity arises from the different distributions of the burning products in both the core and helium shell. In particular, there is a clear trend for faster rise times in the brighter models. This occurs since, in the brighter models, the ${ }^{56} \mathrm{Ni}$-rich core material extends to higher velocities and the opacity of the outermost layers is less owing to the lower masses of the IGEs made in the helium shell detonation. The fainter models show a single, fairly broad UVOIR maximum while the brighter models have an initial peak with a weak shoulder appearing around 30 days after maximum light. This shape is qualitatively similar to that obtained for UVOIR light curves for standard SN Ia models such as W7 (Nomoto et al. 1984); see Fig. 7 of Kromer \& Sim (2009). Full details of our radiative transfer simulations, including complete sets of synthetic light curves and spectra will be presented in a companion study (Kromer et al. 2010, in prep.).

For most of the models (1-4), the $\mathrm{C} / \mathrm{O}$ detonation produces a significant quantity of IMEs $\left(\sim 0.48-0.26 M_{\odot}\right)$, as required to account for the strong lines of e.g. silicon, sulphur and calcium which characterize the maximum-light spectra of SNe Ia. The extreme Model 6, however, makes almost no IMEs and Model 5 yields only a rather small IME mass $\left(\sim 0.1 M_{\odot}\right)$. This means that they are unlikely to be promising candidates to account for real SNe Ia. However, they are still interesting as a demonstration that detonations of such small shell masses can still trigger a secondary detonation in a C/O-WD core. Note that possible initial compositions favoring more oxygen (and neon) for massive WDs (cf. Domínguez et al. 2001; Gil-Pons \& García-Berro 2001) are not considered here. Extrapolating from Seitenzahl et al. (2009), we expect that core detonations would still be triggered for a composition of $30 \%$ carbon and $70 \%$ oxygen (in mass). For compositions with significantly lower carbon fraction like in oxygen/neon WDs detonation criteria are not available yet. Detonation conditions and nucleosynthesis in these stars should be investigated in future studies. The concerns regarding the WD initial composition are alleviated by the fact that our ignition spots are far above the center (see Sect. 4.1 and Table 2) where the carbon fraction is expected to be higher than in the innermost region (see, e.g., Althaus et al. 2005).

The material produced in the helium shell detonation has important observable consequences. Although ${ }^{56} \mathrm{Ni}$ is not very abundant in our models, significant mass-fractions are predicted for the radioactive nuclei ${ }^{52} \mathrm{Fe},{ }^{48} \mathrm{Cr}$, and ${ }^{44} \mathrm{Ti}$. All of these could play a part in powering the supernova light curve (cf. also Bildsten et al. 2007, who consider single helium detonation supernova light curves). The yields of these nuclei are given in Table 3 and, for Model 1, are as large as $20 \%$ of the ${ }^{56} \mathrm{Ni}$ mass. The total mass of all ${ }^{56} \mathrm{Ni},{ }^{52} \mathrm{Fe}$, and ${ }^{48} \mathrm{Cr}$ contributions is given as $M_{L}$ in Table 3.

${ }^{52} \mathrm{Fe}$ and ${ }^{48} \mathrm{Cr}$ have relatively short decay times and release a similar amount of energy per decay as ${ }^{56} \mathrm{Ni}$. Since they are located in the outer ejecta (see Fig. 3), they can contribute to the early phase light curve of the models in which they are abundant. This is illustrated in Fig. 8 where, for Model 1, we compare the light curve computed including the energy released by ${ }^{52} \mathrm{Fe}$ and ${ }^{48} \mathrm{Cr}$ to that obtained if these decay chains are neglected. Generally, the contribution from ${ }^{52} \mathrm{Fe}$ and ${ }^{48} \mathrm{Cr}$ decays is fairly small and is most significant during the rising phase, as expected. The light curve at maximum is completely dominated by energy released from ${ }^{56} \mathrm{Ni}$ and ${ }^{56} \mathrm{Co}$ decays and it remains so throughout the decay phase. There is a modest enhancement around 30 days (of about $\leq 0.2 \mathrm{mag}$ ) that is mostly due to energy produced by ${ }^{48} \mathrm{~V}$, the daughter of ${ }^{48} \mathrm{Cr}$ for which the decay time is $\tau_{1 / 2}=16$ days. The half-life of ${ }^{44} \mathrm{Ti}$ is too long for its decay to directly contribute to the early light curve. Nevertheless, the abundance of titanium is crucial for $U$ - and $B$-band light curves and spectra since even small amounts contribute significantly to the opacity of the shell.

The high velocity IGEs produced in the helium detonation impose an important constraint on the ability of our models to reproduce the early spectra of observed SNe Ia. SN 1991bglike objects show titanium in their spectra, but our models are too bright to fit their light curves. Normal SNe Ia do not show clear signatures of IGEs at early epochs. Similarly, although there is evidence of IGEs affecting the pre-maximum spectra of SN 1991T-like explosions (e.g. Mazzali et al. 1995), these are inconsistent with our models since the important features there are associated with iron rather than the IGEs predicted to be abundant from our nucleosynthesis calculations 9 . However, to fully address the issue of whether the presence of heavy elements created during minimum helium-shell detonations is in contradiction with observations requires detailed radiative transfer simulations and consideration of possible uncertainties in the helium-shell nucleosynthesis. In particular, we would like to emphasize that the nucleosynthetic outcome of the helium detonation depends on the enrichment of the shell with light $\alpha$ nuclei and ${ }^{14} \mathrm{~N}$ (cf. Shen \& Bildsten 2009). Thus, the resulting amounts of ${ }^{52} \mathrm{Fe},{ }^{48} \mathrm{Cr}$, and ${ }^{44} \mathrm{Ti}$ could be significantly lower than found in this study. This will be discussed in a future study (Kromer et al. 2010 , in prep.).

\footnotetext{
${ }^{9}$ Note that the decay-time of ${ }^{52} \mathrm{Fe}$ is so short, $\sim 0.5$ days, that it is expected to have almost completely decayed to ${ }^{52} \mathrm{Cr}$ before the light curve is bright.
} 
M. Fink et al.: Double-detonation sub-Chandrasekhar supernovae: can minimum helium shell masses detonate the core?

\section{Summary}

Motivated by the robustness of a secondary core detonation that was found in a previous study (Paper I), the double-detonation sub-Chandrasekhar scenario for SNe Ia was re-investigated. This time we studied the case of minimum shell masses that might detonate, as calculated by Bildsten et al. (2007) - this is the most conservative case with regard to the question of whether secondary core detonations will be triggered.

In order to improve the accuracy of the explosion dynamics over that of Paper I, we switched to a more realistic detonation prescription in our numerical hydrodynamics simulations, including an energy release that was calibrated with a large nuclear network. The same network was used in a post-processing step to calculate more detailed nucleosynthetic yields.

Even for shell masses as low as $0.0035 M_{\odot}$ and with more realistic helium detonation physics (not producing pure ${ }^{56} \mathrm{Ni}$ as in Paper I) a successful core detonation was found to be very robust (for a discussion of uncertainties concerning the detonation criteria see Sects. 2.2 and 4.1). Thus we predict that the “.Ia" SNe of Bildsten et al. (2007) turn into brighter candidates for "proper" SNe Ia. Given the robustness of the core detonation and the fact that these events might be frequent enough to explain a significant part of the SN Ia rate, the double-detonation subChandrasekhar scenario is a promising candidate for explaining some of these thermonuclear explosions.

Acknowledgements. We want to thank Lars Bildsten and Ken Shen for kindly providing the data for our initial models and for helpful discussions. The simulations presented here were carried out at the Computer Center of the Max Planck Society, Garching, Germany. This work was supported by the Deutsche Forschungsgemeinschaft via the Transregional Collaborative Research Center TRR 33 "The Dark Universe" and the Emmy Noether Program (RO 3676/1-1) of the German Research Foundation.

\section{Appendix A: Detonation tables}

In Paper I we assumed a certain transition density between burning to NSE and incomplete burning in the $\mathrm{C} / \mathrm{O}$ detonation. Additionally, burning was stopped below a roughly estimated fuel density. Therefore the total amount of IGEs and IMEs produced was relatively uncertain. For this work we "calibrated" our burning using a large nuclear network (see Sect. 3.3) in an iteration of explosion simulations and post-processing steps: we set up detonations spherically expanding from the center of a WD star and placed a large number of tracer particles along one axis. As starting point, the production of pure ${ }^{56} \mathrm{Ni}$ at all fuel densities was assumed. The density and temperature profiles attained in this way were then used to determine the detailed nucleosynthetic yields in a post-processing step (see Sect. 3.3) with the large network. Thus the abundances of our five species (or the respective $Q$-values) could be tabulated against the initial density of the unburned matter $\rho_{\mathrm{u}}$. This result was then used in another hydrodynamic simulation of the detonation and a second, more accurate abundance table was calculated from the post-processing. After several such iterations a self-consistent solution was reached. The resulting table (see Fig. A.1) is used in all the simulations presented in Sect. 4 and ensures a consistent energy release and molecular weight in the hydrodynamic simulations. Analogously a table for detonations in pure helium was determined (Fig. A.2). Although our calibration procedure does not account for potential changes in the detonation strength in realistic simulations, it still provides a reasonable approximation. This was confirmed by post-processing our simulations

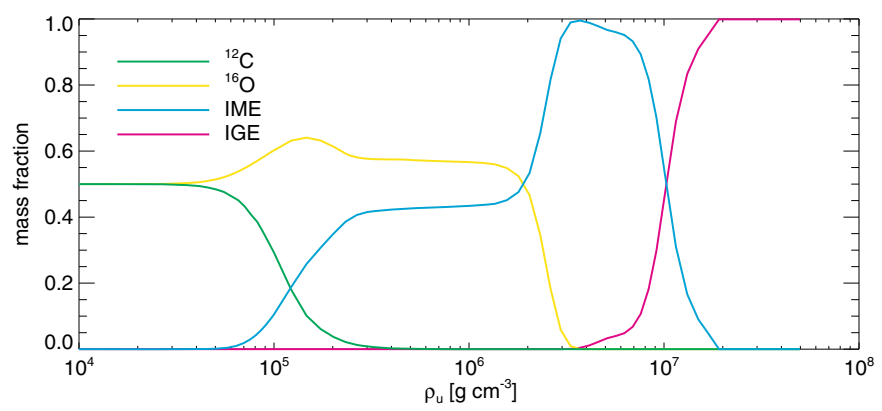

Fig. A.1. Mass fractions of the species in the $\mathrm{C} / \mathrm{O}$ detonation table plotted against the density of the unburned fuel $\rho_{\mathrm{u}}$.

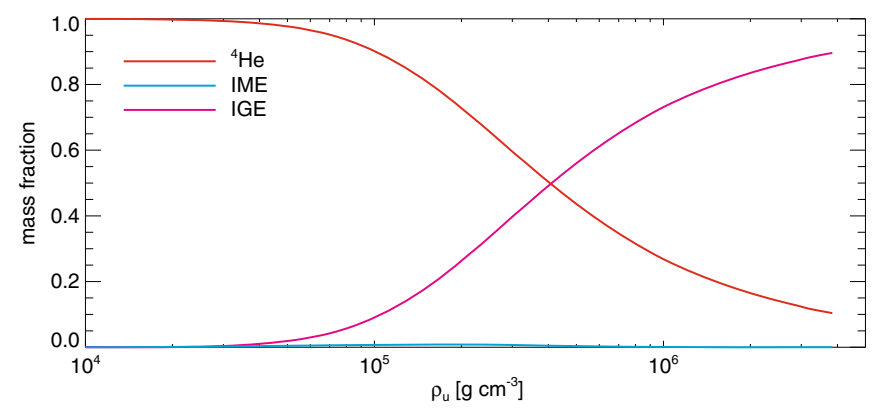

Fig. A.2. Mass fractions of the species in the helium detonation table against $\rho_{\mathrm{u}} \cdot{ }^{12} \mathrm{C}$ and ${ }^{16} \mathrm{O}$ abundances are not shown, as their values are too close to zero.

presented in Sect. 4, which gave results consistent with the hydrodynamic explosion simulations.

In the $\mathrm{C} / \mathrm{O}$ detonation (Fig. A.1) all major burning phases are visible: carbon burning, oxygen burning, and silicon burning. Above densities of about $2 \times 10^{7} \mathrm{~g} \mathrm{~cm}^{-3}$ NSE is reached. NSE is represented only by IGEs in this table. A better representation of NSE, a temperature and density dependent mixture of ${ }^{56} \mathrm{Ni}$ and ${ }^{4} \mathrm{He}$, is calculated in a different module of the code. Helium burning (Fig. A.2) produces mainly IGEs and only a small amount of IMEs at the low densities in our shells $\left(<10^{6} \mathrm{~g} \mathrm{~cm}^{-3}\right)$. A high fraction of the initial helium is left unburned.

\section{References}

Althaus, L. G., Serenelli, A. M., Panei, J. A., et al. 2005, A\&A, 435, 631 Belczynski, K., Bulik, T., \& Ruiter, A. J. 2005, ApJ, 629, 915 Bildsten, L., Shen, K. J., Weinberg, N. N., \& Nelemans, G. 2007, ApJ, 662, L95 Colella, P., \& Woodward, P. R. 1984, J. Comput. Phys., 54, 174

Colgate, S. A., \& McKee, C. 1969, ApJ, 157, 623

Deloye, C. J., Bildsten, L., \& Nelemans, G. 2005, ApJ, 624, 934 Domínguez, I., Höflich, P., \& Straniero, O. 2001, ApJ, 557, 279

Filippenko, A. V., Richmond, M. W., Matheson, T., et al. 1992, ApJ, 384, L15 Fink, M., Hillebrandt, W., \& Röpke, F. K. 2007, A\&A, 476, 1133

Fryxell, B. A., Müller, E., \& Arnett, W. D. 1989, Hydrodynamics and nuclear burning, MPA Green Report 449, Max-Planck-Institut für Astrophysik, Garching

Gamezo, V. N., Wheeler, J. C., Khokhlov, A. M., \& Oran, E. S. 1999, ApJ, 512, 827

Gil-Pons, P., \& García-Berro, E. 2001, A\&A, 375, 87

Golombek, I., \& Niemeyer, J. C. 2005, A\&A, 438, 611

Guderley, G. 1942, Luftfahrtforschung, 19, 302

Hillebrandt, W., \& Niemeyer, J. C. 2000, ARA\&A, 38, 191

Hoyle, F., \& Fowler, W. A. 1960, ApJ, 132, 565

Iwamoto, K., Brachwitz, F., Nomoto, K., et al. 1999, ApJS, 125, 439

Khokhlov, A. M. 1984, Sov. Astron. Lett., 10, 123

Khokhlov, A. M. 1989, MNRAS, 239, 785 
A\&A 514, A53 (2010)

Kromer, M. \& Sim, S. A. 2009, MNRAS, 398, 1809

Kuchner, M. J., Kirshner, R. P., Pinto, P. A., \& Leibundgut, B. 1994, ApJ, 426, L89

Landau, L. D., \& Lifschitz, E. M. 1991, Lehrbuch der theoretischen Physik, Hydrodynamik (Berlin: Akademie Verlag), 6

Langanke, K., \& Martinez-Pinedo, G. 2000, Nucl. Phys. A, 673, 481

Leibundgut, B., Kirshner, R. P., Phillips, M. M., et al. 1993, AJ, 105, 301

Livne, E., \& Arnett, D. 1995, ApJ, 452, 62

Livne, E., \& Glasner, A. S. 1990, ApJ, 361, 244

Mazzali, P. A., Danziger, I. J., \& Turatto, M. 1995, A\&A, 297, 509

Niemeyer, J. C., \& Woosley, S. E. 1997, ApJ, 475, 740

Nomoto, K., Thielemann, F.-K., \& Yokoi, K. 1984, ApJ, 286, 644

Osher, S., \& Sethian, J. A. 1988, J. Comput. Phys., 79, 12

Phillips, M. M., Wells, L. A., Suntzeff, N. B., et al. 1992, AJ, 103, 1632

Ponchaut, N. F., Hornung, H. G., Pullin, D. I., \& Mouton, C. A. 2006, J. Fluid Mech., 560, 103

Reinecke, M., Hillebrandt, W., Niemeyer, J. C., Klein, R., \& Gröbl, A. 1999, A\&A, 347, 724

Reinecke, M., Hillebrandt, W., \& Niemeyer, J. C. 2002a, A\&A, 386, 936
Reinecke, M., Hillebrandt, W., \& Niemeyer, J. C. 2002b, A\&A, 391, 1167

Röpke, F. K. 2005, A\&A, 432, 969

Röpke, F. K., \& Hillebrandt, W. 2005, A\&A, 431, 635

Röpke, F. K., Hillebrandt, W., Niemeyer, J. C., \& Woosley, S. E. 2006, A\&A, 448, 1

Röpke, F. K., Woosley, S. E., \& Hillebrandt, W. 2007, ApJ, 660, 1344

Ruiter, A. J., Belczynski, K., \& Fryer, C. 2009, ApJ, 699, 2026

Seitenzahl, I. R., Meakin, C. A., Townsley, D. M., Lamb, D. Q., \& Truran, J. W. 2009, ApJ, 696, 515

Sharpe, G. J. 1999, MNRAS, 310, 1039

Shen, K. J., \& Bildsten, L. 2009, ApJ, 699, 1365

Sim, S. A. 2007, MNRAS, 375, 154

Stritzinger, M., Leibundgut, B., Walch, S., \& Contardo, G. 2006, A\&A, 450, 241

Thielemann, F.-K., Hashimoto, M.-A., \& Nomoto, K. 1990, ApJ, 349, 222

Thielemann, F.-K., Nomoto, K., \& Hashimoto, M.-A. 1996, ApJ, 460, 408

Travaglio, C., Hillebrandt, W., Reinecke, M., \& Thielemann, F.-K. 2004, A\&A, 425,1029

Truran, J. W., Arnett, W. D., \& Cameron, A. G. W. 1967, Can. J. Phys., 45, 2315 Woosley, S. E., \& Weaver, T. A. 1994, ApJ, 423, 371 